\title{
Magnetic penetration depth and $T_{c}$ in superconducting nickelates
}

\author{
F. Bernardini, ${ }^{1}$ V. Olevano,${ }^{2}$ and A. Cano $\odot^{2}$ \\ ${ }^{1}$ Dipartimento di Fisica, Università di Cagliari, IT-09042 Monserrato, Italy \\ ${ }^{2}$ CNRS \& UGA, Institut Néel, 38042 Grenoble, France
}

(Received 30 October 2019; revised manuscript received 21 January 2020; accepted 4 February 2020; published 27 February 2020)

\begin{abstract}
We compute the nominal magnetic penetration depth of $R \mathrm{NiO}_{2}(R=\mathrm{La}, \mathrm{Nd})$ from first-principles calculations and discuss the results in relation to the superconducting $T_{c}$. We find a marked discrepancy with the wellestablished phenomenology that correlates these two quantities in cuprates (Uemura plot). We also consider the two-dimensional ultrathin limit and estimate the maximum attainable $T_{c}$ to be $\sim 180 \mathrm{~K}$ according to the Nelson-Kosterliz universal relation between the superfluid density and the transition temperature.
\end{abstract}

DOI: 10.1103/PhysRevResearch.2.013219

\section{INTRODUCTION}

The recent discovery of superconductivity in Sr-doped $\mathrm{NdNiO} / \mathrm{SrTiO}_{3}$ thin films [1] has attracted an instantaneous research attention (see, e.g., [2-13]). After many attempts, this can potentially be the first successful extension of high- $T_{c}$ cuprate superconductivity to isostructural/isoelectronic nickelates. This important breakthrough can thus shed light on their elusive microscopic superconducting mechanism, even if it poses some important challenges to current paradigms. In particular, the parent nickelates are metals without magnetic order as opposed to their cuprate counterparts that are antiferromagnetic charge-transfer insulators. Consequently, Cooper pairing in $(\mathrm{Nd}, \mathrm{Sr}) \mathrm{NiO}_{2}$ seems to emerge from a rather different normal nonsuperconducting state.

First-principles calculations based on density-functional theory (DFT) consistently find that, among the five Ni$3 d$ bands, only the $3 d_{x^{2}-y^{2}}$ states intercept the Fermi level $[2-6,14]$. This establishes a promising analogy to $\mathrm{CaCuO}_{2}$, i.e., the parent compound of high- $T_{c}$ cuprates. In fact, the electron-phonon coupling has been ruled out as the exclusive origin of the observed superconductivity in $(\mathrm{Nd}, \mathrm{Sr}) \mathrm{NiO}_{2}$ [5]. At the same time, electrons in the Nd layer make additional electron pockets in the Fermi surface that likely prevent the system from being a simple Mott insulator, with Kondo physics potentially playing a role [6-8]. Besides, the chargetransfer gap between the $\mathrm{Ni}-3 d$ states and $\mathrm{O}-2 p$ states is larger than that in cuprates [9]. On the other hand, spin fluctuations may still be important for superconductivity, even if there is no long-range magnetic order. In that case, the dominant pairing has been proposed to yield a $d$-wave superconducting gap $[3,4]$ with a distinct spin resonance feature that can be tested experimentally [10]. Here, we compute the nominal

Published by the American Physical Society under the terms of the Creative Commons Attribution 4.0 International license. Further distribution of this work must maintain attribution to the author(s) and the published article's title, journal citation, and DOI. magnetic penetration depth of the superconducting nickelates as a function of hole doping and discuss these theoretical results in relation to the observed $T_{c}$.

\section{PRELIMINARIES}

\section{A. Zero-temperature magnetic penetration depth as a band-structure property}

The magnetic penetration depth $\lambda$ is pivotal to explain the Meissner effect and therefore is a fundamental quantity of superconductors [15]. This quantity can be determined experimentally by means of different complementary techniques such as the tunnel diode oscillator technique $[16,17]$ and muon-spin resonance $(\mu \mathrm{SR})$ [18-21]. The temperature dependence of $\lambda$ maps the amount of excited quasiparticles and thereby the structure of the superconducting gap. However, in the London approximation, the zero-temperature magnetic penetration depth in the clean limit reduces to $\lambda_{L}(T=0)=$ $\sqrt{\frac{m^{*}}{\mu_{0} n_{s} e^{* 2}}}$, where $m^{*}$ is the effective mass of charge carriers, $\mu_{0}$ is the vacuum permeability, $n_{s}$ is the charge carrier density, and $e^{*}$ is an effective electron charge. Thus, it basically becomes a band-structure property formally unrelated to the gap function. In fact, from a semiclassical generalization of the London equation [22] or Eilenberger's formulation of superconductivity [23] (see Appendix A), a band-structurespecific result can be obtained as

$$
\left(\lambda^{2}\right)_{i j}^{-1}(T=0)=\frac{\mu_{0} e^{2}}{4 \pi^{3} \hbar} \oint_{\mathrm{FS}} d S \frac{v_{F i} v_{F j}}{v_{F}},
$$

where the integral is over the Fermi surface with $\mathbf{v}_{F}$ being the Fermi velocity (the subscripts $i, j=x, y, z$ refer to principal axes). In the following, we will make use of this result to discuss the superconducting properties of the nickelates.

\section{B. Computational methods}

We computed Eq. (1) from DFT calculations that conveniently reproduce the reported band structure of the $\mathrm{La}$ and $\mathrm{Nd}$ nickelates. Specifically, we used the FLAPW method as 


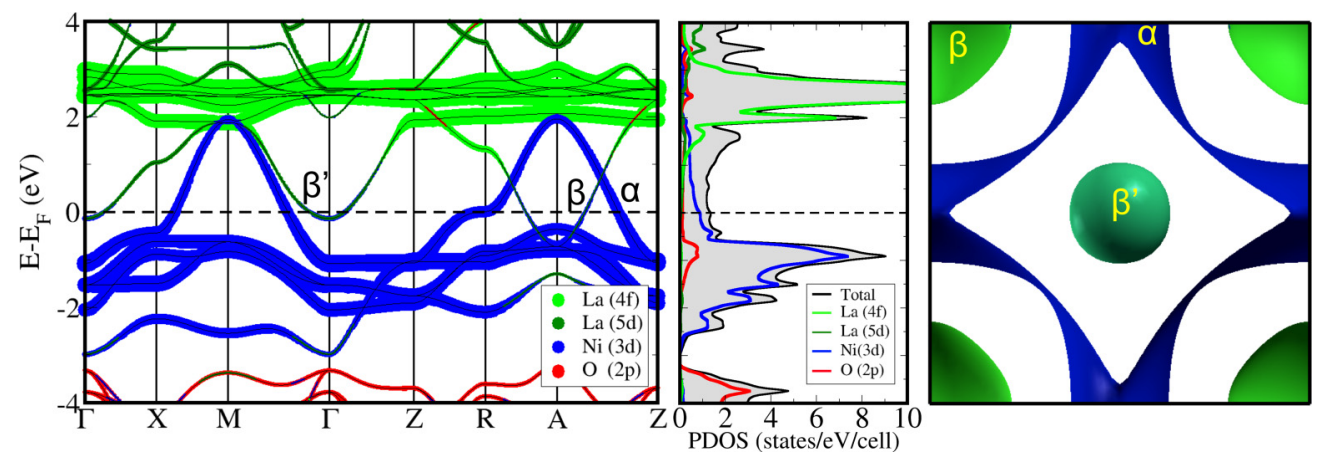

FIG. 1. First-principles band structure (left, "fatband" plot), orbital resolved density of states (middle), and top view of Fermi surface (right) of $\mathrm{LaNiO}_{2}$.

implemented in the WIEN2K package [24] with the local density approximation (LDA) exchange-correlation functional [25]. In order to avoid the ambiguous treatment of the $f$ orbital bands, we followed [2,3] and focused on the mother compound $\mathrm{LaNiO}_{2}$. Further, we modeled $\mathrm{Sr}$ doping as a rigid shift of the Fermi level as in [4] and also within the virtual-crystal approximation (VCA) as in [3]. We also considered $\mathrm{NdNiO}_{2}$ with the Nd- $4 f$ states in the core as well as the influence of epitaxial strain, with which we obtained almost identical results. We performed spinless calculations with muffin-tin radii of $2.5,2.1$, and 1.62 a.u. for the $\mathrm{La}$ $(\mathrm{Nd}), \mathrm{Ni}$, and $\mathrm{O}$ atoms, respectively, and a plane-wave cutoff $R_{\mathrm{MT}} K_{\max }=7.0$. The integration over the Brillouin zone was performed using a $11 \times 11 \times 14 k$ mesh for the self-consistent calculations, while a dense $48 \times 48 \times 48 k$ mesh was used to compute and study the Fermi surface. In our calculations, the Fermi velocity is directly obtained from the expectation value of the momentum operator $\mathbf{p}\left(\mathbf{v}_{F}=\mathbf{p}_{F} / m\right)$, and the dense $k$ mesh was used to further perform the Fermi-surface integral.

\section{RESULTS}

Figure 1 illustrates the reference band structure of the infinite-layer nickelates $[2-4,14,27]$. The Ni-3d states intercepting the Fermi level give rise to the large holelike Fermi surface $\alpha$ dominated by $3 d_{x^{2}-y^{2}}$ contributions. Interestingly, this pocket $\alpha$ seems to originate from two different bands that undergo an avoided crossing along the $Z-R$ path where their $d_{x^{2}-y^{2}}$ and $d_{z^{2}}$ characters swap (see Appendix B). To the best of our knowledge, this feature was first pointed out in [28]. We find that this avoided crossing and its concomitant $d_{x^{2}-y^{2}}-d_{z^{2}}$ mix changes with doping (see Appendix B), thereby having a potential effect on the superconducting instability [29] that has not been considered so far. In addition, there is a self-doping effect due to La- $5 d$ states that results into to the extra electronlike Fermi surfaces $\beta$ and $\beta^{\prime}$.

The nominal $\lambda(0)$ of the superconducting nickelates as a function of $\mathrm{Sr}$ doping is reported in Table I. These values are obtained using a rigid shift of the Fermi level, and essentially the same is obtained using VCA (see Appendix C). Even if superconductivity has been reported for $20 \%$ doping so far, the nominal values computed for other dopings allow us to get an idea of the possible variations in $\lambda(0)$ due to physical changes in the corresponding band structure (which can be taken as a sort of "error bar"). These results confirm that $(\mathrm{Nd}, \mathrm{Sr}) \mathrm{NiO}_{2}$ is a type-II superconductor (i.e., $\kappa \equiv \lambda / \xi>$ $1 / \sqrt{2}$, with $\xi=3.25 \mathrm{~nm}$ being the Ginzburg-Landau coherence length [1]). In the case of the overall in-plane component $\lambda_{x}(0)$, the main contribution originates from the main hole pocket $\alpha$ and does not vary dramatically with doping. The outof-plane component $\lambda_{z}(0)$, in contrast, is initially dominated by the electron pocket $\beta$ and therefore undergoes a more substantial change as $\beta$ shrinks with doping. Note that, despite the apparent two-dimensional (2D) character of $\alpha$ [14], the anisotropy of this contribution is moderate compared to that in

TABLE I. Zero-temperature magnetic penetration length obtained from DFT calculations in the London approximation for different values of $\mathrm{Sr}$ (hole) doping (modeled as a rigid shift of the Fermi level). Only the diagonal terms are nonzero by symmetry, and are denoted by a single subscript $\left(\lambda_{x}=\lambda_{y}\right.$ and $\left.\lambda_{z}\right)$. The effective lambda is defined as $\lambda_{\text {eff }}=3^{1 / 4}\left[1+2\left(\lambda_{x} / \lambda_{z}\right)^{2}\right]^{-1 / 4} \lambda_{x}$, as probed by $\mu \mathrm{SR}$ in polycrystalline samples [26]. The values for $\mathrm{NdNiO}_{2}$ are obtained assuming the Nd- $4 f$ states in the core.

\begin{tabular}{|c|c|c|c|c|}
\hline & Doping & FS & $\left(\lambda_{x}(0), \lambda_{z}(0)\right)(\mathrm{nm})$ & $\lambda_{\text {eff }}(0)(\mathrm{nm})$ \\
\hline \multirow[t]{16}{*}{$\mathrm{LaNiO}_{2}$} & 0 & $\alpha$ & $(47,245)$ & 61 \\
\hline & & $\beta$ & $(140,120)$ & 133 \\
\hline & & $\beta^{\prime}$ & $(365,300)$ & 340 \\
\hline & & Total & $(44,101)$ & 54 \\
\hline & 0.1 & $\alpha$ & $(49,170)$ & 62 \\
\hline & & $\beta$ & $(165,140)$ & 156 \\
\hline & & Total & $(47,108)$ & 57 \\
\hline & 0.2 & $\alpha$ & $(50,215)$ & 64 \\
\hline & & $\beta$ & $(205,175)$ & 194 \\
\hline & & Total & $(48,136)$ & 60 \\
\hline & 0.3 & $\alpha$ & $(51,160)$ & 64 \\
\hline & & $\beta$ & $(275,240)$ & 262 \\
\hline & & Total & $(50,133)$ & 62 \\
\hline & 0.4 & $\alpha$ & $(54,163)$ & 68 \\
\hline & & $\beta$ & $(450,580)$ & 486 \\
\hline & & Total & $(54,156)$ & 67 \\
\hline \multirow[t]{4}{*}{$\mathrm{NdNiO}_{2}$} & 0.2 & $\alpha$ & $(49,180)$ & 62 \\
\hline & & $\beta$ & $(190,160)$ & 179 \\
\hline & & $\beta^{\prime}$ & $(290,220)$ & 262 \\
\hline & & Total & $(47,105)$ & 57 \\
\hline
\end{tabular}




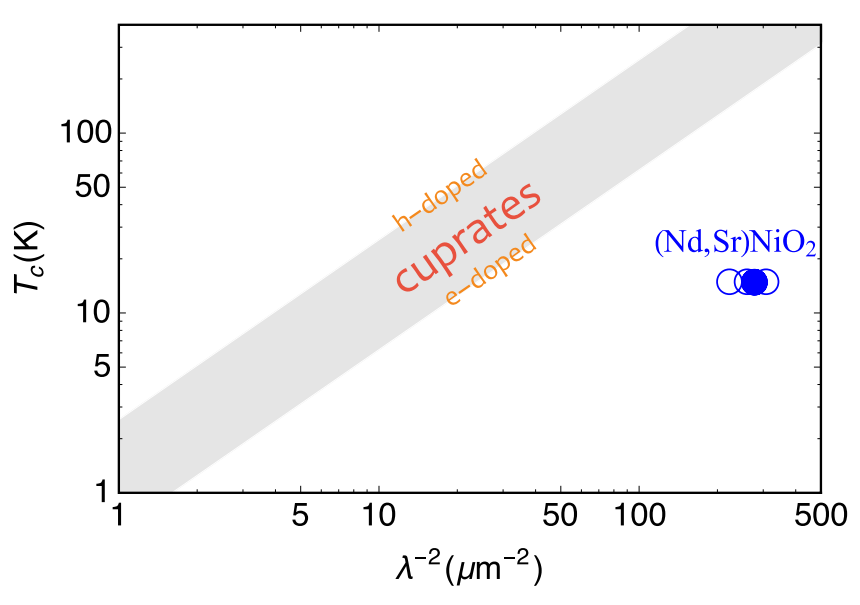

FIG. 2. Uemura log-log plot for the zero-temperature effective magnetic penetration depth of $(\mathrm{Nd}, \mathrm{Sr}) \mathrm{NiO}_{2}$ computed from Eq. (1). The solid circle corresponds to $20 \% \mathrm{Sr}$ doping and $T_{c}=15 \mathrm{~K}$, while the open circles are associated to the $10 \%, 30 \%$, and $40 \%$ dopings in Table I assuming the same $T_{c}$.

cuprates and displays a nonmonotonous behavior with doping. This should not be surprising if the $\alpha$ pocket results from two different bands undergoing an avoided crossing that changes with doping (see Fig. 3 in Appendix B). Beyond that, the effective $\lambda_{\text {eff }}$ probed by $\mu$ SR turns out to be essentially that of the hole pocket $\alpha$ (with $\beta$ having the effect of reducing the anisotropy resulting from $\alpha$ only).

\section{DISCUSSION}

Our results are summarized in Fig. 2 in a Uemura plot. As we can see, the calculated $\lambda_{\text {eff }}$ 's are totally off the expected values for a cuprate with $T_{c} \approx 15 \mathrm{~K}$ (shaded region). The modification of the effective mass due to correlations can to some extent improve the agreement. However, the mass has to be $>10$ times larger to do the job, which seems unlikely according to DMFT results (see, e.g. [13]).

We note that a presumably related discrepancy has been pointed out for the Hall resistance of the parent compound [2]. Specifically, the Hall coefficient is inconsistent with the large holelike Fermi surface $\alpha$. To reproduce the sign of the measured coefficient and obtain a fair agreement with its numerical value, a gapped $\alpha$ pocket has to be assumed. Analogously, in the case of the magnetic penetration depth, the cuprate trend in the Uemura plot is regained if the $\alpha$ contribution is neglected and only the electron pocket $\beta$ is taken account for $\lambda(0)$ at $20 \%$ Sr doping. Even if the Hall coefficient and $\lambda(0)$ are both a measure of the DOS, one would tend to think that is rather fortuitous coincidence. In any case, this illustrates that reconciling these estimates with the well-established phenomenology of cuprates seems to require a rather significant modification of the corresponding band structure (this, or the system is to be understood as a failed room-temperature superconductor according to its $\lambda$ ).

Alternatively, superconductivity in $(\mathrm{Nd}, \mathrm{Sr}) \mathrm{NiO}_{2}$ thin films can be a $2 \mathrm{D}$ phenomenon and hence a different rationale must be applied. This will be naturally the case in the ultrathin limit, and will also be relevant if superconductivity is even- tually driven by the interface with the substrate [30,31]. The magnetic penetration depth, being a measure of the superfluid density, is also related to the superfluid stiffness $D_{s}$. This relation can be exploited to set bounds on the superconducting transition temperature since fluctuations of the phase of the superconducting order parameter will be the ultimate limiting factor in 2D [32]. Such a bound directly reads from NelsonKosterlitz universal jump of the superfluid density [33]:

$$
k_{B} T_{c} \leqslant \pi D_{s} / 2 .
$$

The 2D superfluid stifness can be estimated from our previous calculations as $D_{s} \approx \frac{\hbar^{2}}{4 \mu_{0} e^{2}} \frac{d}{2 \pi} \lambda_{x}^{-2}$, where $d$ is the interlayer spacing (i.e., the $c$ lattice parameter). This gives a maximum $T_{c}$ of about $145 \mathrm{~K}$. By restricting the integral (1) to the $k_{F z}=0$ line of the three-dimensional (3D) Fermi surface this value increases to $180 \mathrm{~K}$. While formally rigorous, this estimate has to be understood as a rather conservative upper bound since the superfluid density at $T=0$, and hence the corresponding stiffness, can reasonably be assumed to overestimate that at $T_{c}$.

\section{CONCLUSIONS}

In summary, we have computed the zero-temperature magnetic penetration depth $\lambda(0)$ of the newly superconducting nickelate $\mathrm{NdNiO}_{2}$ relying on first-principles DFT calculations to fully take into account its band-structure specific features. $\lambda(0)$ is a fundamental descriptor of superconductivity displaying a phenomenological correlation to $T_{c}$ in cuprates and in other unconventional superconductors. Our calculations confirm the system as a type-II superconductor. The in-plane component of $\lambda(0)$ is found to be dominated by the hole Fermi-surface pocket and no substantial change is obtained with doping. However, the extra electron pocket has a nonnegligible impact on the eventual anisotropy. Remarkably, the nominal $\lambda(0)$ and the reported $T_{c}$ do not follow the same correlation observed in the cuprates. If the same correlation were to apply, $\mathrm{NdNiO}_{2}$ would be a room-temperature superconductor. This suggests that either the reported band structure needs to be revisited or the superconducting nature of nickelates is different. In the 2D case relevant for the ultrathin limit and/or if the actual phenomenon corresponds to interfacial superconductivity [30,31], the maximum attainable $T_{c}$ is estimated to be $\sim 180 \mathrm{~K}$ from the Nelson-Kosterlitz universal jump of the superfluid density.

\section{ACKNOWLEDGMENT}

F.B. acknowledges the Visiting Scientist Program of the Centre de Physique Theorique de Grenoble-Alpes (CPTGA) for financial support.

\section{APPENDIX A: DERIVATION OF EQ. (1).}

Here, we outline the derivation of Eq. (1) within Eilenberger's formulation of superconductivity. This derivation can be found in more detail in [17,23], for example. Eilenberger's picture is obtained directly from Gor'kov equations after integrating out fast-varying degrees of freedom [34]. Thus, the 
current density reads as

$$
\mathbf{j}=-4 \pi e k_{B} T \operatorname{Im} \sum_{\omega>0}\left\langle\mathbf{v}_{F} g\right\rangle,
$$

where $g$ is the Eilenberger function associated to normal excitations. Here, $\omega=(2 n+1) \pi k_{B} T / \hbar$ are Matsubara frequencies and the average is defined such that $\langle X\rangle=\int \frac{d^{2} k_{F}}{(2 \pi)^{3} \hbar v_{F}} X$. In the clean case, Eq. (A1) for the current is supplemented by the set of equations

$$
\begin{gathered}
\left(\hbar \mathbf{v}_{F} \cdot \boldsymbol{\Pi}+2 \hbar \omega\right) f=2 \Delta g, \\
\left(-\hbar \mathbf{v}_{F} \cdot \boldsymbol{\Pi}^{*}+2 \hbar \omega\right) f^{+}=2 \Delta^{*} g, \\
\Delta\left(\mathbf{r}, \mathbf{v}_{F}\right)=2 \pi k_{B} T \sum_{\omega>0}^{\omega_{\max }}\left\langle V\left(\mathbf{v}_{F}, \mathbf{v}_{F}^{\prime}\right) f\left(\mathbf{r}, \omega, \mathbf{v}_{F}^{\prime}\right)\right\rangle_{\mathbf{v}_{F}^{\prime}},
\end{gathered}
$$

where $\boldsymbol{\Pi}=\boldsymbol{\nabla}-\frac{2 i e}{\hbar} \mathbf{A}$. Here $\Delta$ and $V$ represent the gap function and the effective coupling, respectively, while the functions $f$ and $f^{+}$describe the superconducting condensate and are such that $f f^{+}=1-g^{2}$. In the absence of currents and fields, the ground-state uniform solution of Eilenberger equations reads as $f_{0}=f_{0}^{+}=\Delta_{0} /\left(\Delta_{0}^{2}+\hbar^{2} \omega^{2}\right)^{1 / 2}$, $g_{0}=\hbar \omega /\left(\Delta_{0}^{2}+\hbar^{2} \omega^{2}\right)^{1 / 2}$. The presence of weak fields and supercurrents can be treated perturbatively to obtain $g=g_{0}+$ $g_{1}$, where the correction $g_{1}$ reads as $g_{1}=i \hbar \frac{\Delta_{0}^{2}}{2\left(\Delta_{0}^{2}+\hbar^{2} \omega^{2}\right)^{3 / 2}} \mathbf{v}_{F}$. $\left(\nabla \theta+\frac{2 e}{\hbar} \mathbf{A}\right)$, with $\theta$ being the overall phase acquired by $\Delta$, $f$, and $f^{+}$. Substituting this in Eq. (1) yields the London relation $j_{i}=-\frac{1}{\mu_{0}}\left(\lambda^{2}\right)_{i j}^{-1} a_{j}$ between the current and the "gaugeinvariant vector potential" $\mathbf{a}=\frac{\hbar}{2 e} \boldsymbol{\nabla} \theta+\mathbf{A}$, where

$$
\left(\lambda^{2}\right)_{i j}^{-1}=4 \pi \mu_{0} e^{2} k_{B} T \sum_{\omega>0}\left\langle\frac{\Delta_{0}^{2} v_{F i} v_{F j}}{\left(\Delta_{0}^{2}+\hbar^{2} \omega^{2}\right)^{3 / 2}}\right\rangle .
$$

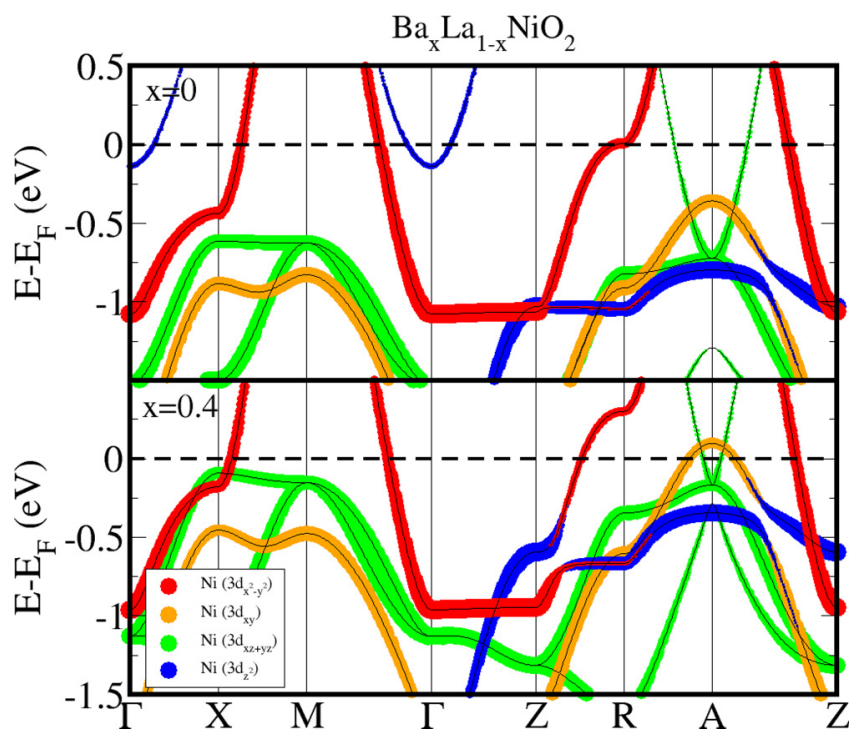

FIG. 3. Zoom of the electronic band structure of $\mathrm{LaNiO}_{2}$ (top) and $\mathrm{La}_{0.6} \mathrm{Ba}_{0.4} \mathrm{NiO}_{2}$ within VCA (bottom) in a "fatband" plot.

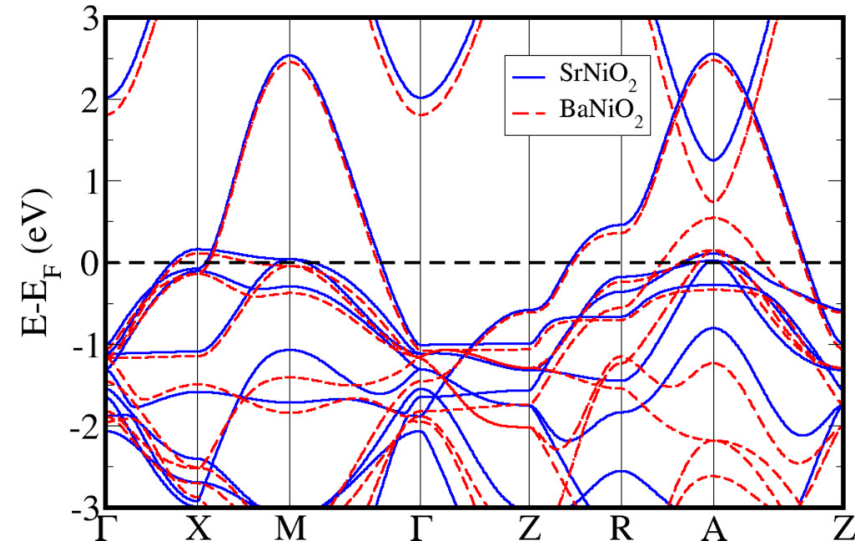

FIG. 4. Band structure of $\mathrm{SrNiO}_{2}$ and $\mathrm{BaNiO}_{2}$ compared.

In the $T=0$ limit,

$$
\begin{aligned}
\left(\lambda^{2}\right)_{i j}^{-1}(T=0) & =2 \mu_{0} e^{2} \hbar\left\langle v_{F i} v_{F j} \int_{0}^{\infty} d \omega \frac{\Delta_{0}^{2}}{\left(\Delta_{0}^{2}+\hbar^{2} \omega^{2}\right)^{3 / 2}}\right\rangle \\
& =2 \mu_{0} e^{2} \hbar\left\langle\left. v_{F i} v_{F j} \frac{\omega}{\left(\Delta_{0}^{2}+\hbar^{2} \omega^{2}\right)^{1 / 2}}\right|_{0} ^{\infty}\right\rangle \\
& =2 \mu_{0} e^{2}\left\langle v_{F i} v_{F j}\right\rangle,
\end{aligned}
$$

which corresponds to Eq. (1).

\section{APPENDIX B: AVOIDED CROSSING AND $d_{x^{2}-y^{2}}-d_{z^{2}}$ MIX}

Figure 3 shows a zoom of the electronic band structure of $(\mathrm{La}, \mathrm{Ba}) \mathrm{NiO}_{2}$ near the Fermi level. As we can see, the Ni-3d states generating the holelike pocket in the Fermi surface ( $\alpha$ in Fig. 1) appears to be associated to two different bands. These bands undergo an avoided crossing along the $Z-R$ path that changes with doping. Thus, the apparent $2 \mathrm{D} \alpha$ pocket is in reality a $3 \mathrm{D}$ one by its very nature.

TABLE II. Zero-temperature magnetic penetration length obtained from DFT calculations in the London approximation for different values of $\mathrm{Ba}$ (hole) doping (modeled using the VCA). The effective lambda is defined as $\lambda_{\text {eff }}=3^{1 / 4}\left[1+2\left(\lambda_{x} / \lambda_{z}\right)^{2}\right]^{-1 / 4} \lambda_{x}$, as probed by $\mu \mathrm{SR}$ in polycrystalline samples [26].

\begin{tabular}{lccc}
\hline \hline $\mathrm{La}_{1-x} \mathrm{Ba}_{x} \mathrm{NiO}_{2}$ & FS & $\left(\lambda_{x}(0), \lambda_{z}(0)\right)(\mathrm{nm})$ & $\lambda_{\text {eff }}(0)(\mathrm{nm})$ \\
\hline$x=0.1$ & $\alpha$ & $(49,168)$ & 62 \\
& $\beta$ & $(164,145)$ & 157 \\
& Total & $(47,110)$ & 57 \\
$x=0.2$ & $\alpha$ & $(50,153)$ & 63 \\
& $\beta$ & $(210,197)$ & 205 \\
& Total & $(49,121)$ & 60 \\
$x=0.3$ & $\alpha$ & $(51,156)$ & 64 \\
& $\beta$ & $(284,285)$ & 284 \\
& Total & $(50,138)$ & 62 \\
$x=0.4$ & $\alpha$ & $(52,163)$ & 65 \\
& $\beta$ & $(345,400)$ & 361 \\
& Total & $(52,151)$ & 65 \\
\hline \hline
\end{tabular}




\section{APPENDIX C: VCA CALCULATIONS}

Here, we report additional calculations using the virtualcrystal approximation (VCA) and comment on this approach for the infinite-layer nickelates. We restrict ourselves to the mother compound $\mathrm{LaNiO}_{2}$ and consider the substitution of $\mathrm{La}$ with $\mathrm{Ba}$ instead of $\mathrm{Sr}$ as in [3]. Thus, we consider the $\mathrm{La}_{1-x} \mathrm{Ba}_{x} \mathrm{NiO}_{2}$ material which is further modeled by a virtual compund $X \mathrm{NiO}_{2}$ where the $X$ atom has a nuclear charge $Z=$ $57-x$. We note that this strategy would be perfect if the band structure of $\mathrm{SrNiO}_{2}$ and $\mathrm{BaNiO}_{2}$ were identical. However, this is not exactly the case as can be seen in Fig. 4. In particular, the dispersion of the bands is noticeably different at the $A$ point which leads to non-negligible differences in the Fermi surface. This will be more important for the case of electron doping. Despite of such a difference, the values of $\lambda(0)$ calculated using the VCA are essentially the same as those calculated with the rigid band approximation (see Table II). In addition, the two methods yield the exactly same trend as a function of doping: while both $\lambda_{x}$ and the $\beta$ contribution to $\lambda_{z}$ increase with doping, the $\alpha$ contribution to $\lambda_{z}$ displays a nonmonotonous behavior. The agreement obtained using these two different approaches thus strengthens the validity of our results.
[1] D. Li, K. Lee, B. Y. Wang, M. Osada, S. Crossley, H. R. Lee, Y. Cui, Y. Hikita, and H. Y. Hwang, Nature (London) 572, 624 (2019).

[2] A. S. Botana and M. R. Norman, Phys. Rev. X 10, 011024 (2020).

[3] H. Sakakibara, H. Usui, K. Suzuki, T. Kotani, H. Aoki, and K. Kuroki, arXiv: 1909.00060.

[4] X. Wu, D. D. Sante, T. Schwemmer, W. Hanke, H. Y. Hwang, S. Raghu, and R. Thomale, arXiv:1909.03015.

[5] Y. Nomura, M. Hirayama, T. Tadano, Y. Yoshimoto, K. Nakamura, and R. Arita, Phys. Rev. B 100, 205138 (2019).

[6] M. Hepting et al., Nat. Mater. (to be published) (2020), doi: 10.1038/s41563-019-0585-Z.

[7] G.-M. Zhang, Y.-F. Yang, and F.-C. Zhang, Phys. Rev. B 101, 020501 (2020).

[8] Y.-H. Zhang and A. Vishwanath, arXiv:1909.12865.

[9] M. Jiang, M. Berciu, and G. A. Sawatzky, arXiv:1909.02557.

[10] T. Zhou, Y. Gao, and Z. D. Wang, arXiv:1910.05757.

[11] J. E. Hirsch and F. Marsiglio, Physica C (Amsterdam) 566, 1353534 (2019).

[12] L.-H. Hu and C. Wu, Phys. Rev. Research 1, 032046 (2019).

[13] S. Ryee, H. Yoon, T. J. Kim, M. Y. Jeong, and M. J. Han, arXiv: 1909.05824.

[14] K.-W. Lee and W. E. Pickett, Phys. Rev. B 70, 165109 (2004).

[15] M. Tinkham, Introduction to Superconductivity (Dover, New York, 2004).

[16] R. Prozorov and R. W. Giannetta, Supercond. Sci. Technol. 19, R41 (2006).

[17] R. Prozorov and V. G. Kogan, Rep. Prog. Phys. 74, 124505 (2011).

[18] Y. J. Uemura, G. M. Luke, B. J. Sternlieb, J. H. Brewer, J. F. Carolan, W. N. Hardy, R. Kadono, J. R. Kempton,
R. F. Kiefl, S. R. Kreitzman et al., Phys. Rev. Lett. 62, 2317 (1989).

[19] A. Amato, Rev. Mod. Phys. 69, 1119 (1997).

[20] J. E. Sonier, J. H. Brewer, and R. F. Kiefl, Rev. Mod. Phys. 72 , 769 (2000).

[21] A. Bhattacharyya, D. T. Adroja, M. Smidman, and V. K. Anand, Sci. China: Phys., Mech. Astron. 61, 127402 (2018).

[22] B. S. Chandrasekhar and D. Einzel, Ann. Phys. 505, 535 (1993).

[23] V. G. Kogan, Phys. Rev. B 66, 020509(R) (2002).

[24] P. Blaha, K. Schwarz, G. Madsen, D. Kvasnicka, J. Luitz, R. Laskowski, F. Tran, and L. D. Marks, WIEN2k, An Augmented Plane Wave + Local Orbitals Program for Calculating Crystal Properties (Karlheinz Schwarz, Techn. Universität Wien, Austria, 2018).

[25] J. P. Perdew and A. Zunger, Phys. Rev. B 23, 5048 (1981).

[26] V. Fesenko, V. Gorbunov, and V. Smilga, Physica C (Amsterdam) 176, 551 (1991).

[27] V. I. Anisimov, D. Bukhvalov, and T. M. Rice, Phys. Rev. B 59 , 7901 (1999).

[28] F. Bernardini, V. Olevano, X. Blase, and A. Cano, arXiv: 1911.11788

[29] H. Sakakibara, H. Usui, K. Kuroki, R. Arita, and H. Aoki, Phys. Rev. B 85, 064501 (2012).

[30] Q. Li, C. He, J. Si, X. Zhu, Y. Zhang, and H.-H. Wen, arXiv:1911.02420.

[31] F. Bernardini and A. Cano, arXiv:2001.02133.

[32] T. Hazra, N. Verma, and M. Randeria, Phys. Rev. X 9, 031049 (2019).

[33] D. R. Nelson and J. M. Kosterlitz, Phys. Rev. Lett. 39, 1201 (1977).

[34] G. Eilenberger, Z. Phys. A 214, 195 (1968). 